\title{
MEASUREMENT AND SIMULATION OF SPACE-CHARGE DEPENDENT TUNE SEPARATION IN FNAL BOOSTER
}

Daniel McCarron (IIT, Chicago, Illinois), James Frederick Amundson, William Pellico, Panagiotis Spentzouris, Raymond E. Tomlin (Fermilab, Batavia, Illinois), Linda Klamp Spentzouris (Illinois Institute of Technology, Chicago, Illinois.

\section{Abstract}

In recent years, a number of space-charge studies have been performed in the FNAL Booster. The Booster is the lowest energy circular accelerator in the Fermilab chain of machines, with an injection energy of $400 \mathrm{MeV}$. Measurement and simulation of space charge coupling in the Booster will be presented. The coupling measurement was performed using a standard technique, albeit repeated for different injected beam intensities. The initial transverse tune separation was minimized $(\mathrm{Qx}=\mathrm{Qy}=6.7)$, followed by a systematic skew quadrupole strength variation. Transverse beam oscillation frequencies were recorded while exciting the beam. These frequencies were recorded for a range of $1.0 \times 10^{12}$ to $3.5 \times 10^{12}$ particles. A linear increase in the measured tune separation with beam intensity was observed. For comparison, beam coupling was also simulated with the space-charge code Synergia [1]. This code has successfully modeled the space-charge tune shift in the Booster [2], and compares favorably to other space charge codes and analytic results.

\section{INTRODUCTION}

In this paper we examine the 2-D transverse-oscillatory behavior of a particle beam under the influence of spacecharge self forces, i.e. transverse-plane coupling, in the FNAL Booster. We consider first a beam of weakly interacting particles which will undergo transverse oscillations according to

$$
\frac{d^{2}(x, y)}{d s^{2}}+\left(\frac{v_{(x, y)_{0}}}{R}\right)^{2}(x, y)=0
$$

where $v_{0}$ is the number of oscillations per turn in the horizontal or vertical direction (betatron tune), $\mathrm{R}$ is the accelerator radius, and $\mathrm{s}$ is the longitudinal coordinate. The elements responsible for the restoring force(s) in (1), are the vertically- and horizontally-focusing quadrupoles, whose respective fields are given by

$$
B_{x}=\frac{\partial B_{x}}{\partial y} y, \quad B_{y}=\frac{\partial B_{y}}{\partial x} x
$$

The transverse oscillation frequencies depend on the size of the (uniform) field gradients, and the spacing of these quadrupoles. In a perfect, linear system, the horizontal and vertical motions are independent (uncoupled), and forever remain so. In reality, even in the linear case, such is never the case. Errors or misalignments in quadrupole fields, image-charge wall effects, and space-charge can change the value of these tunes and couple the horizontal motion to the vertical. We analyze the dependence on space charge in particular. Should a focusing quadrupole be misaligned by an angle $\phi$, the fields in (2) transform according to

$$
\begin{aligned}
& B_{y}=\frac{\partial B_{y}}{\partial x}[x \cos 2 \phi+y \sin 2 \phi], \\
& B_{x}=\frac{\partial B_{x}}{\partial y}[y \cos 2 \phi-x \sin 2 \phi]
\end{aligned}
$$

Skew-quadrupoles $\left(\phi= \pm 45^{\circ}\right)$ are conventionally employed to "trim" out this effect. Coupling from sources other than quadrupole misalignments, however, cannot be corrected in this way. In what follows we outline a method for determining the dependence of transverse coupling on intensity.

A particle beam in the presence of linear restoring forces and coupling will have normal mode tunes $v_{ \pm}$given by

$$
v_{ \pm}^{2}=\frac{1}{2}\left[v_{x}^{2}+v_{y}^{2} \pm \sqrt{\left(v_{x}^{2}-v_{y}^{2}\right)^{2}+4 q^{4}}\right]
$$

where $\mathrm{q}$ is the strength of the coupling between the horizontal and vertical planes. We consider the degenerate case, where the $v_{x}=v_{y}=v_{0}$, for which (4) reduces to

$$
v_{ \pm}^{2}=\left[v_{0}^{2} \pm q^{2}\right]
$$

If the coupling strength depended solely on the skewquadrupole fields, one could obtain uncoupled operation by scanning the skew-quadrupole currents, and locating the intersection of the normal-mode tunes plotted as a function of the field strength near this degenerate condition. In practice, coupling may never be eliminated, but can be obtained from the minimal separation between these tunes. In the following figure this is demonstrated for a particular intensity $\left(1.0 \times 10^{12}\right.$ particles). 


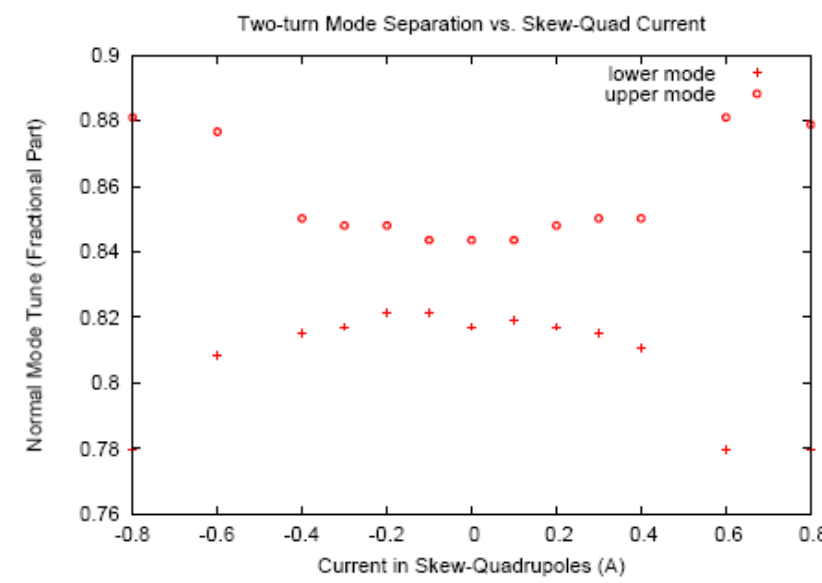

Figure 1: Normal-mode tunes under varying skewquadrupole current for intensity of $1.0 \times 10^{12}$ particles. Coupling minimum corresponds to the effect of intensity.

\section{EXPERIMENTAL RESULTS}

The mode separation represents the coupling aside from skew-quadrupole fields. We have performed these scans for a range of intensities in the FNAL Booster from 2 turns injected $\left(1.0 \times 10^{12}\right.$ particles) to 8 turns injected $\left(3.1 \times 10^{12}\right.$ particles), as shown in Figure 2.

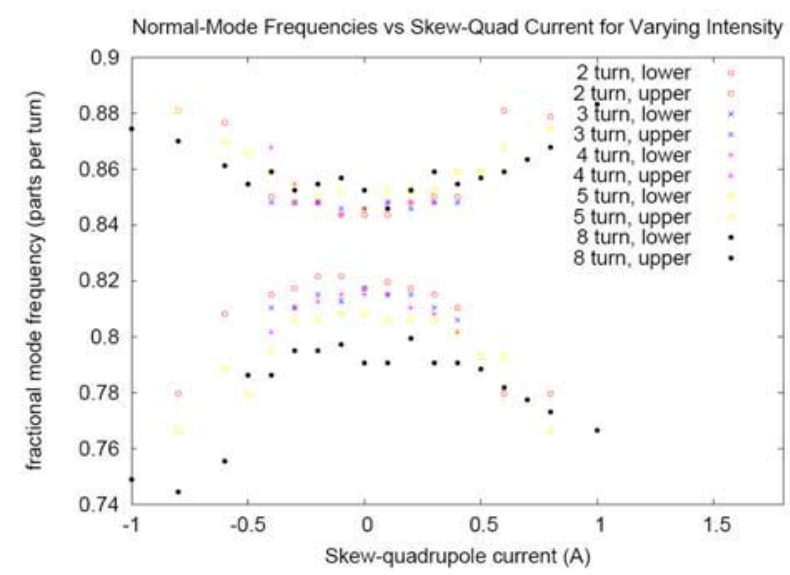

Figure 2: Upper and lower normal-mode tunes under varying skew-quadrupole current

As alluded to above, intensity was not linear with the number of injected turns since some particles were lost, especially at 8 turns injected, where upwards of 35\% remained uncaptured. Accounting for this, intensity dependence of this coupling strength is shown in Figure 3.
Mode separation as a function of intensity



Figure 3: Normal-mode tune separation vs. intensity

\section{SIMULATION RESULTS}

Conditions from experiment have been simulated using the parallel code Synergia. Using similar settings for horizontal and vertical tune, we show the effect of varying skew-quadrupole current in the limit of zero intensity (Figure 4).

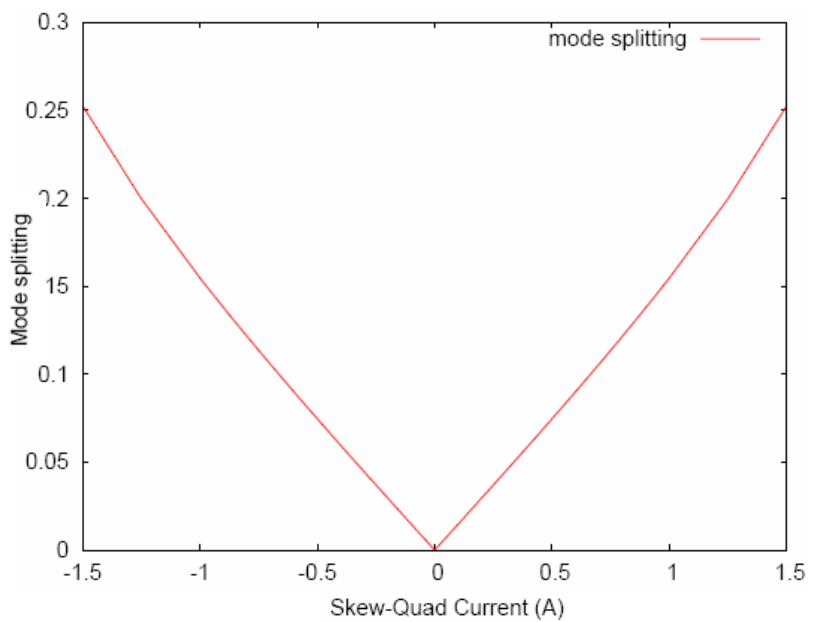

Figure 4: Normal-Mode splitting as a function of skewquadrupole current.

At 1A typical mode separation is around 0.1 in data and 0.15 in simulation. In trying to simulate the intensity, less success was achieved, and we are currently attempting to duplicate this effect in simulation.

Problems with obtaining the intensity effect on mode splitting are not surprising. While our measurement is unambiguous it is nonetheless peculiar. One would not expect the space-charge self-force to affect the coherent motion of the beam, at least not measurably at the BPM's, so the mechanism for this space-charge coupling is currently under investigation. 
1. P. Spentzouris, J. Amundson. Synergia: A 3D Accelerator Modelling Tool with 3D Space Charge. Journal of Computational Physics, Volume 211, Issue 1,1 January 2006, Pages 229248

2. P. Spentzouris, J. Amundson. FNAL Booster: experiment and modeling. By FERMILAB-CONF03-127, Jun 2003. Presented at Particle Accelerator Conference (PAC 03), Portland, Oregon, 12-16 May 2003 